\title{
Swelling of polymer networks with topological constraints: Application of the Helmis-Heinrich-Straube model
}

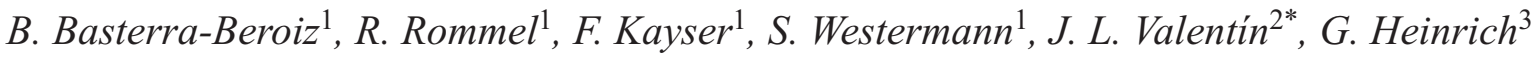 \\ ${ }^{1}$ Goodyear Innovation Center Luxembourg, Avenue Gordon Smith, L-7750 Colmar-Berg, Luxembourg \\ ${ }^{2}$ Instituto de Ciencia y Tecnología de Polímeros (CSIC), c/ Juan de la Cierva 3, 28006 Madrid, Spain \\ ${ }^{3}$ Leibniz-Institut für Polymerforschung Dresden e.V., Hohe Straße 6, D-01069 Dresden, Germany
}

Received 18 January 2018; accepted in revised form 19 March 2018

\begin{abstract}
For the first time since its formulation in 1986, the theoretical approach proposed by Helmis, Heinrich and Straube (HHS model), which considers the contribution of topological restrictions from entanglements to the swelling of polymer networks, is applied to experimental data. The main aspects and key equations are reviewed and their application is illustrated for unfilled rubber compounds. The HHS model is based on real networks and gives new perspectives to the interpretation of experimental swelling data for which the entanglement contributions are usually neglected by considering phantom network models. This investigation applies a reliable constrained-chain approach through a deformation-dependent tube model for defining the elastic contribution of swollen networks, which is one of the main limitations on the applicability of classical (affine) Flory-Rehner and (non-affine) phantom models. This short communication intends to provide a baseline for the application and validation of this modern approach for a broader class of rubber materials.
\end{abstract}

Keywords: rubber, swelling, entanglements, tube model, cross-link density

\section{Introduction}

The determination of the average cross-link density of rubber compounds by equilibrium swelling experiments $[1,2]$ is a standard method in rubber science and technology. A relative comparison of the cross-link density can be achieved with different approaches from which then the average mass between cross-links $M_{\mathrm{c}}$ can be calculated; however, the careful selection of the underlying physical models is critical to obtain reliable information on a quantitative level.

The swelling behavior of rubber networks is usually based on the Flory-Rehner model [3-6], which postulates that mixing and elastic components of the free energy are additive and separable. According to the Flory-Huggins approach for the Gibbs free energy of mixing, the related chemical potential for cross- linked polymers, $\Phi_{\text {mix }}$, as a function of the volumetric fraction of rubber $\phi_{\mathrm{r}}$ is written in Equation (1):

$\Phi_{\text {mix }}=\ln \left(1-\phi_{\mathrm{r}}\right)+\phi_{\mathrm{r}}+\chi \cdot \phi_{\mathrm{r}}^{2}$

where $\chi$ is the Flory-Huggins interaction parameter $[7,8] \cdot \chi$ strongly influences the accuracy of swellingderived quantities [9] and is known precisely only for a limited number of polymer-solvent pairs.

The expression of the elasticity contribution to the chemical potential depends on the assumed molecular theory. Thus, the selection of an appropriate physical model represents the highest source of uncertainty in the final calculated cross-link density from equilibrium swelling experiments [9]. Two classical limits exist for the elastic response of swollen networks: The affine Flory-Rehner [4-6] and the

${ }^{*}$ Corresponding author, e-mail: jlvalentin@ictp.csic.es

C BME-PT 
non-affine phantom approach $[10,11]$. These models, which are still the most used nowadays, are based on phantom networks i.e. Gaussian chain statistics with free chain movement without interference from the neighboring chains where the effect of entanglements is not considered.

The main aim of this communication is the analysis of the accuracy of another, not so known, theoretical approach published in 1986 by Helmis, Heinrich and Straube (HHS model) [12] . Here, the elasticity term of real swollen polymer networks also considers the conformational constraints through a deformationdependent network-chain tube model [13], which in general has been identified as a powerful and robust constitutive model [14] in order to understand the elasticity of unswollen rubber materials.

After its initial publication, the HHS approach has never been applied to analyze experimental data. While other constrained-chain approaches have been applied to define the effect of entanglements on the elasticity contribution in swollen networks (e.g. [15]), the consideration of the tube model in the framework of the equilibrium swelling formalism will constitute an advance in consistency for the comparison of experimental data from different methods and the unification of different approaches for the understanding of rubber behavior.

The main assumptions made in the HHS model are the additivity of the mixing and elastic contributions to the free enthalpy for swollen networks, an isotropic deformation caused by swelling and a variation of the elastic free energy that equals that of the elastic free enthalpy, for which three additive terms were provided in the original paper. Under these considerations, the change in the chemical potential related to the elastic expansion of the polymer chains for the equilibrium state according to the HHS model is given by Equation (2):

$\Phi_{\text {elastic }}=\frac{-V_{\mathrm{s}} \cdot \phi_{\mathrm{rm}}^{1 / 3} \cdot G_{\mathrm{c}} \cdot F\left(x_{\mathrm{m}}\right)}{R \cdot T}$

where $V_{\mathrm{s}}$ is the molar volume of the solvent, $\phi_{\mathrm{rm}}$ the volumetric fraction of rubber corresponding to the maximum swelling conditions, $R$ the gas constant, $T$ the temperature and $G_{\mathrm{c}}$ is the cross-link modulus in the dry state, which contains information about the average density of chemical junctions but does not allow for distinguishing their specific nature (e.g. mono-, di- or polysulfidic). $F\left(x_{\mathrm{m}}\right)$ (with $x_{\mathrm{m}} \equiv$ $\left.\phi_{\mathrm{rm}}^{-2 / 3}\right)$ is a factor derived from the free energy of the tube model which depends on the contribution of cross-links and entanglements to the modulus in dry state using $G_{\mathrm{c}}$ and $G_{\mathrm{e}}=G_{\mathrm{N}} / 2$, respectively, and the release of topological constraints as a result of the application of a deformation. The latter is represented by the parameter $\xi$, which is assumed to be equal to the dry state value $\xi_{0}$ varying between 0 (total release corresponding to a phantom network $G=G_{\mathrm{c}}$ ) and $1\left(G=G_{\mathrm{c}}+G_{\mathrm{e}}\right)$. For the calculations with experimental data, $\xi_{0}=1$ will be used in order to consider the contributions of both cross-links and entanglements. The function $F\left(x_{\mathrm{m}}\right)$ is calculated according to Equation (3):

$$
\begin{aligned}
& F\left(x_{\mathrm{m}}\right)=1-3 \cdot \frac{G_{\mathrm{N}}}{G_{\mathrm{c}}} \cdot\left(x_{\mathrm{m}}\right)^{-\frac{1}{2} \cdot\left(5+\xi_{0}\right)} \cdot\left(1+\frac{\xi_{0}}{3}\right)+ \\
& +\left(2 \xi_{0}+6\right) \cdot\left(\frac{G_{\mathrm{N}}}{G_{\mathrm{c}}}\right)^{2} \cdot \frac{\left(x_{\mathrm{m}}\right)^{-\xi_{0}-4}}{1+4 \cdot\left(\frac{G_{\mathrm{N}}}{G_{\mathrm{c}}}\right)^{2} \cdot\left(x_{\mathrm{m}}\right)^{-\xi_{0}-3}}
\end{aligned}
$$

Assuming the additivity of the elastic and mixing free energy contributions [3-6] and considering [16] $G_{\mathrm{c}}=A_{\mathrm{c}} \cdot\left(\rho R T / M_{\mathrm{c})}\right.$ (being $A_{\mathrm{c}}$ the microstructure factor accounting for junction fluctuations and $\rho$ the rubber network density), a general expression for the average molecular weight between cross-links for the equilibrium state (where $\phi_{\mathrm{r}}=\phi_{\mathrm{rm}}$ ) is obtained (Equation (4)):

$M_{\mathrm{c}}=-\frac{V_{\mathrm{s}} \cdot \phi_{\mathrm{r}}^{1 / 3} \cdot A_{\mathrm{c}} \cdot \rho \cdot F\left(x_{\mathrm{m}}\right)}{\ln \left(1-\phi_{\mathrm{r}}\right)+\phi_{\mathrm{r}}+\chi \cdot \phi_{\mathrm{r}}^{2}}$

It can be shown that this general expression naturally includes the classical swelling models when introducing the length scales related to fluctuations of cross-links, $r_{\mathrm{c}}$, and chain segments, $r_{\mathrm{e}},[13]$ and their relation to $A_{\mathrm{c}}$ :

- (Non-affine) phantom model: The limit of free fluctuation of the junctions with [16] $r_{\mathrm{c}}=r_{\mathrm{e}}=\infty$ corresponds to the model of James and Guth [10, 11]. In this model, the end-to-end vectors deform affinely, while fluctuations of cross-links are not affected by the external strain. Therefore, chain vectors deform non-affinely following Equation (5):

$A_{c}=1-\frac{2}{f}$ and $F\left(x_{\mathrm{m}}\right)=1$

where $f$ is the cross-link functionality.

- Flory-Rehner model: It corresponds to an affine model of the phantom network, which assumes that the junction fluctuations are totally suppressed leading to an affine deformation of the end-to-end 
vectors. In this case [16], $r_{\mathrm{c}}=r_{\mathrm{e}}=0$ and $A_{\mathrm{c}}$ and $F\left(x_{\mathrm{m}}\right)$ follow the dependences described in Equation (6):

$$
A_{c}=1 \text { and } F\left(x_{\mathrm{m}}\right)=\left(1-\frac{2 \phi_{\mathrm{r}}^{2 / 3}}{f}\right)=\frac{f-2 x_{\mathrm{m}}^{-1}}{f}
$$

- HHS: This model adopts - beside the presence of entanglements - the picture of restricted junction fluctuations like in the work of Erman and Flory [17]. The microstructure factor [18] $A_{\mathrm{c}}$ depends on the range of cross-link fluctuations, $r_{\mathrm{c}}$, and the root-mean-square distance between two successive cross-links or trapped entanglements, $\left\langle R_{0}^{2}\right\rangle_{\mathrm{ce}}^{1 / 2}$ (Equation (7)):

$$
A_{\mathrm{c}}=\frac{1}{2}+\frac{1}{\pi^{1 / 2}} \cdot \frac{K_{\mathrm{c}} \mathrm{e}^{-K_{\mathrm{c}}^{2}}}{\operatorname{erf}\left(K_{\mathrm{c}}\right)} \text { and } K_{\mathrm{c}}=\sqrt{6} \cdot \frac{r_{\mathrm{c}}}{\left\langle R_{0}^{2}\right\rangle_{\mathrm{ce}}^{1 / 2}}
$$

Assuming that $r_{\mathrm{c}}$ can be identified with the tube radius [16] and expressing the average end-to-end distance of the network chains in terms of $M_{\mathrm{c}}$ (see e.g. [19]), the approximation in Equation (8) in terms of the average number of segments between cross-links $\left(N_{\mathrm{c}}\right)$ and entanglements $\left(N_{\mathrm{e}}\right)$ is expected to hold:

$K_{\mathrm{c}} \approx \sqrt{\frac{3}{2}} \cdot \frac{\sqrt{N_{\mathrm{e}}}}{\sqrt{N_{\mathrm{c}}}}$

Using this formula, the $A_{\mathrm{c}}$ values can be calculated for each compound individually. The value of the function $F\left(x_{\mathrm{m}}\right)$ is calculated following Equation (3) which depends on the moduli in the dry state with $G_{\mathrm{N}}=(2 / \sqrt{6}) \cdot\left(\rho R T / M_{\mathrm{e}}\right), \xi_{0}$ and $\phi_{\mathrm{r}}$. A simplified graphic representation of the relaxation of topological constraints in the swollen state as proposed by the HHS model is shown in Figure 1.

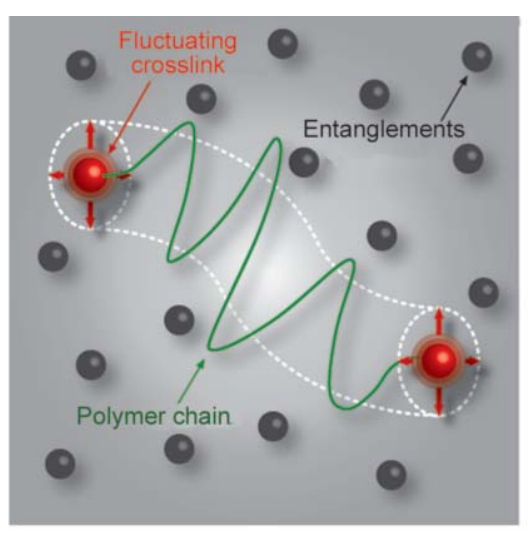

a)

\section{Experimental section}

\subsection{Samples}

Three different rubber matrices, often used in technical applications, have been used in this work: Natural rubber (NR), styrene-butadiene rubber (SBR) and butadiene rubber (BR). In all the cases, the groups of samples contained different levels of curatives, so that variations in cross-link density would be obtained. In addition, the compounds contained other additional ingredients that can appear in technically relevant rubber formulations, such as antidegradants, plasticizers or co-cure accelerators as will be detailed in the following paragraphs.

The recipes of the unfilled NR samples with different amounts of sulfur (between 1 and $2.5 \mathrm{phr}$ ) and CBS ( $N$-cyclohexyl-2-benzothiazolesulfenamide) (between 1 and $3 \mathrm{phr}$ ) were provided in a previous publication [20], where an in-depth assessment of cross-link density by several methods was reported for these samples. The samples contained in addition $2.5 \mathrm{phr}$ of 6-PPD ( $N$-(1,3-dimethylbutyl)- $N$ '-phenyl$p$-phenylenediamine), $3 \mathrm{phr}$ of $\mathrm{ZnO}$ and $1 \mathrm{phr}$ of stearic acid.

Both the SBR and BR samples were mixed in tangential laboratory mixer and contained different amounts of sulfur and CBS, with content ratio of $\mathrm{S}$ to CBS equal to 1 . The rest of the ingredients were kept constant for each group. The following abbreviations are used in Tables 1 and 2: DPD ( $N, N^{\prime}$-diphenylpara-phenylenediamine), DPG (diphenylguanidine) and TDAE (treated distillate aromatic extract type mineral oil). The amount of each ingredient is indicated in phr (parts per hundred rubber i.e. mass units of an ingredient for a hundred mass units of rubber).

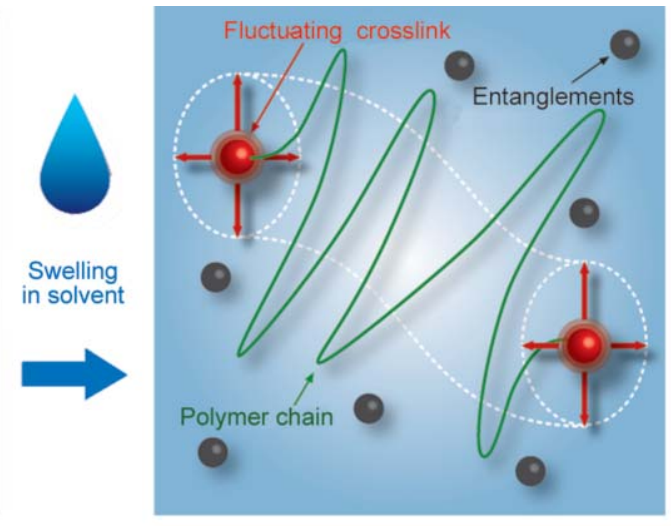

b)

Figure 1. Schematic representation of the relaxation of topological constraints in dry state (a) upon the immersion of the network on a good swelling solvent (b): The virtual tube surrounding the chain becomes wider, and cross-links fluctuate in a broader range. 
Table 1. Formulations for the unfilled SBR compounds (in phr).

\begin{tabular}{|l|r|r|r|r|r|}
\hline \multicolumn{1}{|c|}{ Samples } & \multicolumn{1}{c|}{$\mathbf{3 6 8}$} & \multicolumn{1}{c|}{$\mathbf{3 7 1}$} & \multicolumn{1}{c|}{$\mathbf{3 7 4}$} & \multicolumn{1}{c|}{$\mathbf{3 7 7}$} & \multicolumn{1}{c|}{$\mathbf{3 8 0}$} \\
\hline SBR & 137.5 & 137.5 & 137.5 & 137.5 & 137.5 \\
\hline 6-PPD & 2.5 & 2.5 & 2.5 & 2.5 & 2.5 \\
\hline TDAE Oil & 2.0 & 2.0 & 2.0 & 2.0 & 2.0 \\
\hline Stearic acid & 3.0 & 3.0 & 3.0 & 3.0 & 3.0 \\
\hline DPD & 0.5 & 0.5 & 0.5 & 0.5 & 0.5 \\
\hline ZnO & 2.5 & 2.5 & 2.5 & 2.5 & 2.5 \\
\hline Sulfur & 2.1 & 1.3 & 1.9 & 1.7 & 1.5 \\
\hline CBS & 2.1 & 1.3 & 1.9 & 1.7 & 1.5 \\
\hline DPG & 1.5 & 1.5 & 1.5 & 1.5 & 1.5 \\
\hline
\end{tabular}

Table 2. Formulations for the unfilled BR compounds (in phr).

\begin{tabular}{|l|r|r|r|}
\hline \multicolumn{1}{|c|}{ Samples } & \multicolumn{1}{c|}{$\mathbf{7 4 4}$} & \multicolumn{1}{c|}{$\mathbf{7 4 6}$} & \multicolumn{1}{c|}{$\mathbf{7 4 8}$} \\
\hline BR & 100.00 & 100.00 & 100.00 \\
\hline 6-PPD & 2.50 & 2.50 & 2.50 \\
\hline TDAE oil & 30.17 & 30.17 & 30.17 \\
\hline Stearic acid & 3.00 & 3.00 & 3.00 \\
\hline DPD & 0.50 & 0.50 & 0.50 \\
\hline Sulfur & 1.30 & 1.70 & 2.10 \\
\hline CBS & 1.30 & 1.70 & 2.10 \\
\hline DPG & 1.50 & 1.50 & 1.50 \\
\hline ZnO & 2.50 & 2.50 & 2.50 \\
\hline
\end{tabular}

All samples were cured at $150^{\circ} \mathrm{C}$ up to $t_{90}$ (vulcanization time corresponding to a torque level of $90 \%$ of the maximum torque).

\subsection{Experimental procedure}

The procedures described below correspond to the experimental methods applied for the SBR and BR samples, while the details for NR testing can be found in reference [20].

\subsubsection{Stress-strain experiments and tube model fittings}

Uniaxial stress-strain measurements of S2 dumbbells were carried out on a Zwick tensile tester at a strain rate of $10 \mathrm{~mm} / \mathrm{min}(\sim 50 \% / \mathrm{min}$ for an initial length of $20 \mathrm{~mm}$ ) in order to ensure quasistatic conditions and enable the application of the extended tube model of rubber elasticity for calculation. The test temperature was $23^{\circ} \mathrm{C}$. Fittings of the experimental data to the tube model $[13,25]$ were carried out with a MATLAB programmed tool which included a selfconsistent numerical code for the calculation of the microstructure factor $A_{\mathrm{c}}$. Several fittings were done per sample and the final averages of the cross-link modulus $G_{\mathrm{c}}$ and the entanglement modulus $G_{\mathrm{e}}$ (related to the average mass between entanglements $M_{\mathrm{e}}$ through the expression $\left.G_{\mathrm{e}}=(1 / \sqrt{6}) \cdot\left(\rho R T / M_{\mathrm{e}}\right)\right)$ were further used for the calculations in this paper. The average results for the coefficient of variation (standard deviation multiplied by 100 and divided by the mean value) of $G_{\mathrm{c}}$ and $G_{\mathrm{e}}$ considering different fitting ranges for a set of experimental data and including NR, SBR and BR samples are below 5\%, with individual deviations not exceeding $10 \%$.

\subsubsection{Equilibrium swelling experiments}

The samples were immersed in toluene at $23^{\circ} \mathrm{C}$ during a minimum time of 12 hours. The excess of solvent was dried using blotting paper before the determination of the equilibrium swelling weight of the sample. The samples were dried in an oven at $80^{\circ} \mathrm{C}$ during 2 hours for the determination of the weight after extraction. The reported results are based on the average of two measurements for each sample. The difference with used NR experimental conditions (in which the samples were immersed during 72 hours at $25^{\circ} \mathrm{C}$, drying with blotting paper and then $24 \mathrm{~h}$ at $60^{\circ} \mathrm{C}$ and using four specimens per compound) was motivated by availability criteria since both procedures are assumed to be above the minimum time to reach equilibrium swelling for the analyzed samples and therefore equivalent in practice. The repeatability of the measurements in the described conditions was high (coefficients of variation not exceeding $2 \%$ in general).

The used experimental procedures did not include extraction prior to swelling since it was assumed that low molecular weight components would be extracted by the toluene (extracted fraction are between 30.7 and $31.8 \%$ for the SBR-based compounds and between 24.3 and $24.9 \%$ in the case of the BR samples) and that this previous step might also enhance the extraction of the antioxidants, leading to a higher degradation and oxidation that might influence the measurement.

\section{Results}

As shown in the general equation for the calculation of $M_{\mathrm{c}}$ from swelling experiments Equation (4), the product of the factors $A_{\mathrm{c}}$ and $F\left(x_{\mathrm{m}}\right)$ defines the individual dependences of each physical model. Therefore, the different approaches to describe the elastic 
behavior of rubber networks in swollen state can be compared through the factor $B$, which is defined in Equation (9):

$$
B=A_{\mathrm{c}} \cdot F\left(x_{\mathrm{m}}\right)
$$

The variation of $A_{\mathrm{c}}, F\left(x_{\mathrm{m}}\right)$, their product $B$ and the finally obtained $M_{\mathrm{c}}$ values according to each of the swelling models presented in the Introduction are illustrated in this section. The reported swelling-related calculations have been done following previously published recommendations [9] and using the $\chi$ parameter for the pair NR-toluene $[21]: \chi_{\text {NR-toluene }}=$ $0.427+0.112 \cdot \phi_{\mathrm{r}}^{2}$.

Starting with the microstructure factor $A_{\mathrm{c}}$, Figure 2 shows the two extreme values associated to the oversimplified affine Flory-Rehner and non-affine

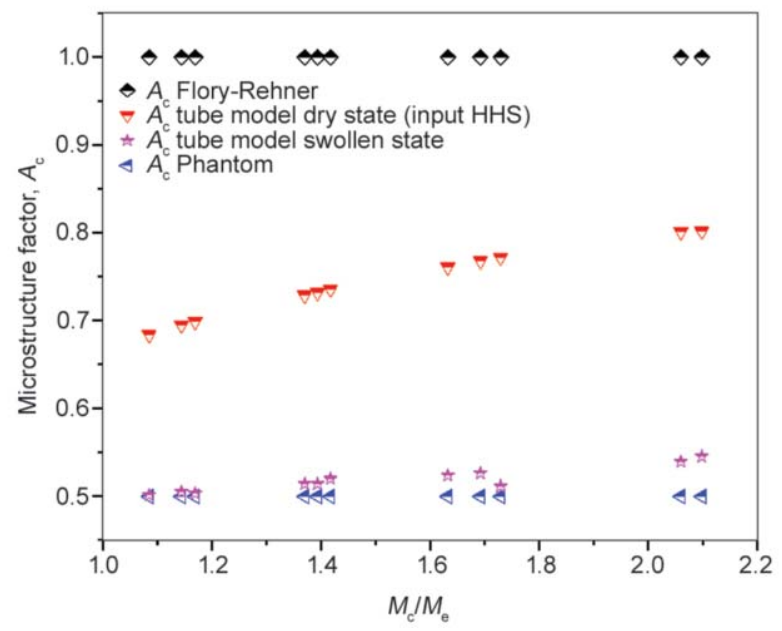

Figure 2. Microstructure factor $A_{\mathrm{c}}$ for unfilled NR samples vulcanized with different $\mathrm{S} / \mathrm{CBS}$ ratios (different $\left.M_{\mathrm{c}} / M_{\mathrm{e}}\right)$.

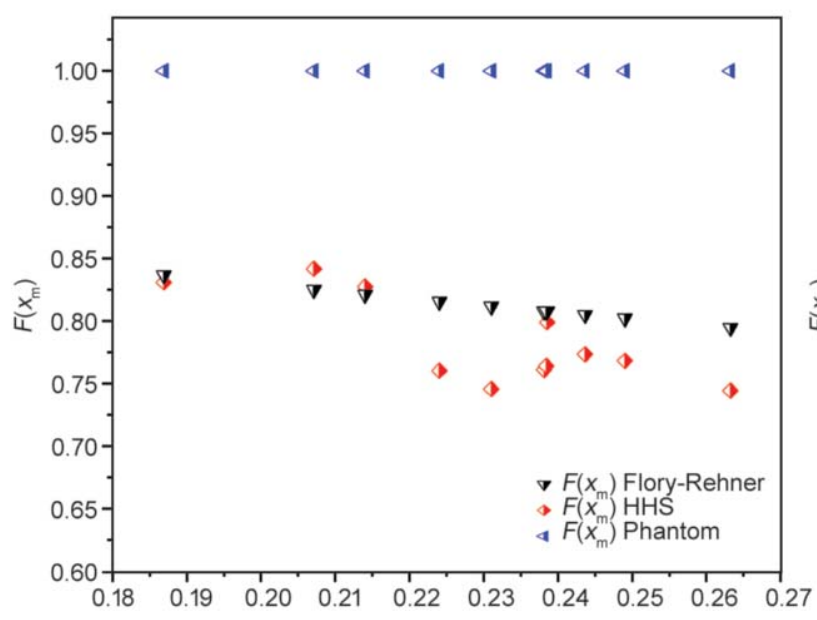

a)

$\phi_{\mathrm{r}}$ phantom models for the series of unfilled NR compounds [20] vulcanized with sulfur and CBS. Opposite to them, the HHS model reflects the increasing restriction of junction fluctuations at higher entanglement densities expected for real networks. In swollen state, the rubber network is deformed and the virtual tube caused by entanglement effects deforms non-affinely [12] as shown in Equation (10):

$d_{\mathrm{sw}}=d_{0} \cdot \boldsymbol{\phi}_{\mathrm{r}}^{-1 / 2}$

where $d_{\mathrm{sw}}$ and $d_{0}$ are the tube radii in swollen and dry state, respectively. The large deformation and the consequent partial release of the packing effects in the swollen samples bring the network behavior near the limit of free fluctuation of the junctions. Nevertheless, it is important to note the appreciable increment of $A_{\mathrm{c}}$ with $M_{\mathrm{c}} / M_{\mathrm{e}}$ ratio, which evidences the importance of taking into account the entanglement effect in the elastic response of swollen samples.

In the case of the function $F\left(x_{\mathrm{m}}\right)$, Figure 3 a shows that it presents a slight decrease with $\phi_{\mathrm{r}}$ for the HHS approach. Although the showed HHS trend is closer to the behavior described by the affine model (being those values below the maximum value of 1 of the non-affine phantom model), it is important to remark that the underlying physical framework for both approaches is different. The values of $F\left(x_{\mathrm{m}}\right)$ for the Flory-Rehner and phantom models depend only on $\phi_{\mathrm{r}}$, while the observed scattering of the HHS model is due to an additional dependence: $F\left(x_{\mathrm{m}}\right)$ varies with $M_{\mathrm{c}} / M_{\mathrm{e}}$ (Figure 3b), showing higher quantitative differences with the Flory-Rehner and phantom models.

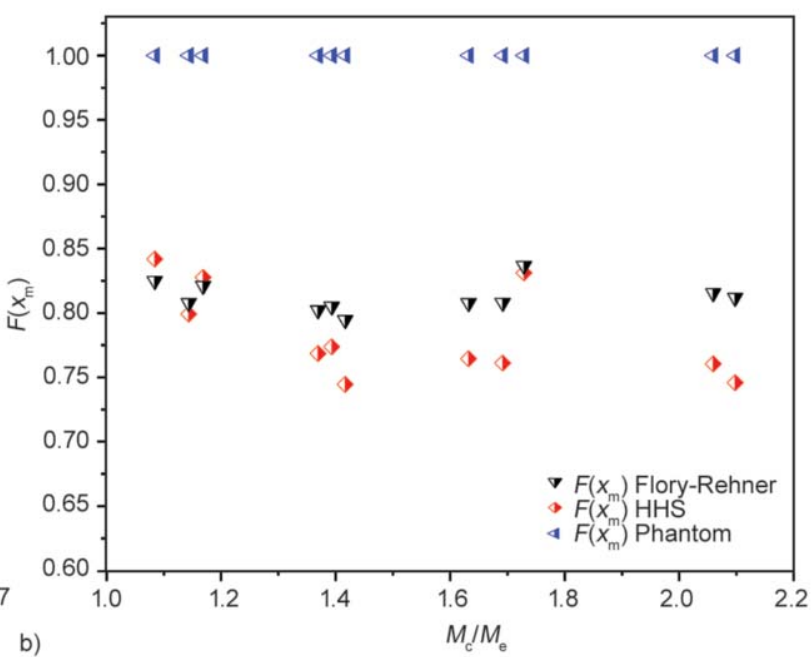

Figure 3. a) $F\left(x_{\mathrm{m}}\right)$ for the Flory-Rehner, HHS and phantom models as a function of the volumetric fraction of rubber $\phi_{\mathrm{r}}$; b) Variation of $F\left(x_{\mathrm{m}}\right)$ in the HHS model with $M_{\mathrm{c}} / M_{\mathrm{e}}$ in comparison to the Flory-Rehner and phantom model results. 


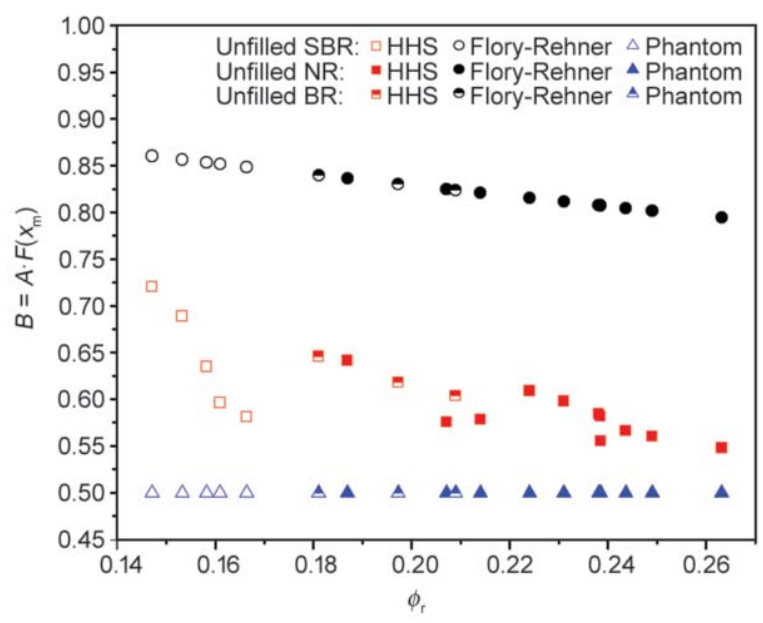

Figure 4. Model-dependent factor $B$ as a function of the volumetric fraction of rubber for unfilled SBR, BR and NR compounds.

The overall effect of the $A_{\mathrm{c}}$ and $F\left(x_{\mathrm{m}}\right)$ factors is shown in Figure 4 through the study of the variation of the model dependent factor $B$ with $\phi_{\mathrm{r}}$ for three different types of unfilled rubbers: NR, SBR and BR. The observed general trend is the same for all the compounds: The HHS model calculations generally tend to approach the phantom model values for increasing $\phi_{\mathrm{r}}$, as presented in the original theoretical paper [12]. Therefore, the deviation between both models needs to be critically considered, especially when analyzing the results of samples with low cross-link densities (related to high $M_{\mathrm{c}} / M_{\mathrm{e}}$ values). It is observed that for the used materials and range of volumetric fractions of rubber, the HHS model is quantitatively closer to the phantom model. Note that the discussion in refs [22-24] favored the use of the phantom model versus the Flory-Rehner model and did not consider the HHS model yet.

An example of the application of the presented HHS model to experimental data is shown in Figure 5, where the $M_{\mathrm{c}}$ results from swelling for the unfilled NR samples are plotted versus $M_{\mathrm{c}}$ from uniaxial quasi-static stress-strain data analyzed with the wellestablished extended tube model of rubber elasticity $[13,25]$. It should be noted that the average mass between cross-links $M_{\mathrm{c}}$ is related to cross-link density $v_{\mathrm{c}}$ through $v_{\mathrm{c}}=\left(\rho N_{\mathrm{A}}\right) / M_{\mathrm{c}}$, where $\rho$ denotes the density and $N_{\mathrm{A}}$ Avogadro's constant i.e. the higher the molecular mass between cross-links, the lower is the density of chemical junctions per unit volume. Figure 5 evidences that the final $M_{\mathrm{c}}$ values obtained by application of the HHS model are the closest results to the line of total correspondence between swelling and mechanical testing (a linear fitting without

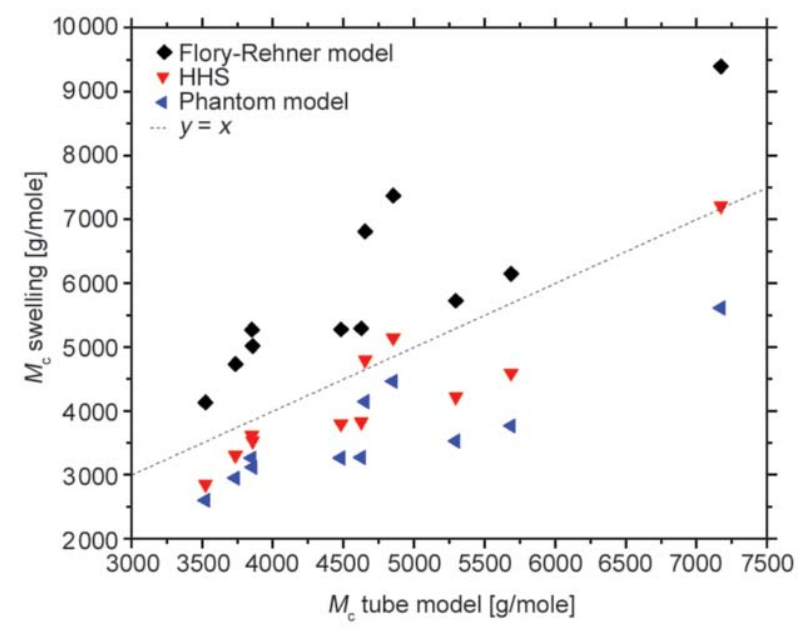

Figure 5. Average mass between cross-links $M_{\mathrm{c}}$ by different swelling models versus the results of tube model fittings of stress-strain curves. The HHS model presents the best quantitative agreement between swelling and mechanical characterization.

intercept has a slope of 0.91 with $R^{2}=0.831$ ) and they are in-between the two limits represented by the classical Flory-Rehner and phantom approaches.

\section{Discussion}

Once a constrained model for the elastic contribution in swollen state has been applied, the observed deviations in Figure 5 for the HHS model (with a maximum difference with respect to the tube model fittings of $20 \%$ ) should be mainly related to experimental variations in both swelling and mechanics and the parameter $\chi$, which reflects the complex network swelling behavior. Consequently, it is proposed to take advantage of the high consistency in the assumptions behind the HHS model and the tube model, in order to combine equilibrium swelling experiments and mechanical tests (tube model stressstrain fitting results as input in Equation (4)) to evaluate the $\chi$ parameter in swollen rubber networks. Using this approach for the described unfilled NR compounds, the obtained results show differences between 0.2 and $8 \%$ from the estimations using the Flory-Huggins function proposed by Horkay et al. [21] (Figure 6). Ideally, if the experimental errors are low enough, it is possible to track the change of $\chi$ as a function of the volumetric fraction of rubber as it is shown for the unfilled SBR samples with $\mathrm{S} / \mathrm{CBS}=1$ and different sulfur levels in Figure 7. Note that in this case, a specific consideration of non-elastic network chain defects has not been considered to correct $M_{\mathrm{c}}$ values from mechanical and swelling tests [26], which leads to an additional dependence on the 


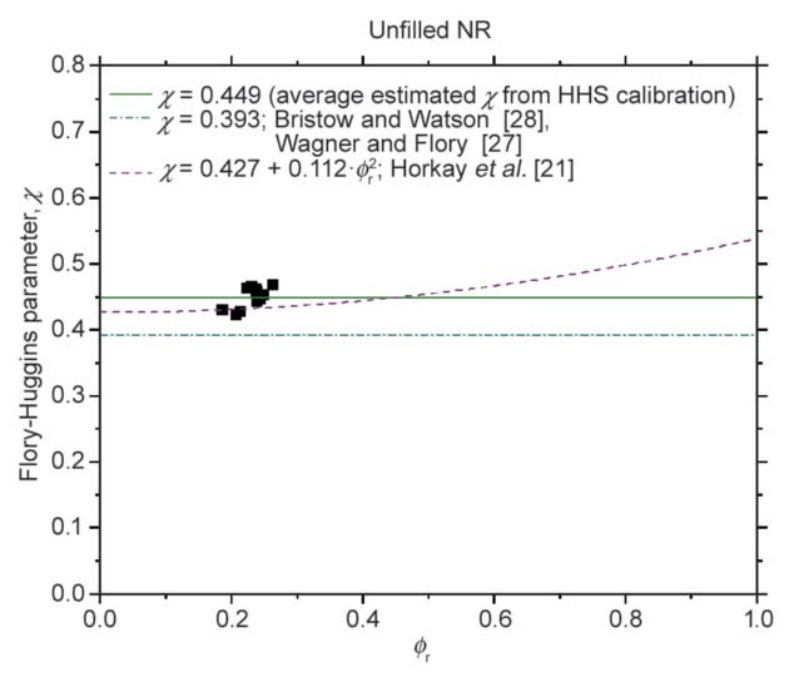

Figure 6. Estimated $\chi$ using the proposed calibration (black points) and comparison with the literature values for the NR-toluene pair: constant [27, 28] and variable [21] with $\phi_{\mathrm{r}}$.

sample preparation and curing (which could be the reason for the lack of clear trends in Figure 6).

The proposed approach constitutes an improvement in the accuracy of the obtained result, given that previous strategies to calculate $\chi$ from calibrations considered the Flory-Rehner result [29] or the phantom model [26]. This calibration procedure is especially interesting for the study of polymer networks with unknown $\chi$ values because it is inexpensive, relatively quick and only requiring the use of standard laboratory devices.

It is important to point out that the reported concentration dependence of $\chi$ for cross-linked polymers $[21,30-32]$ has been related to both the additivity

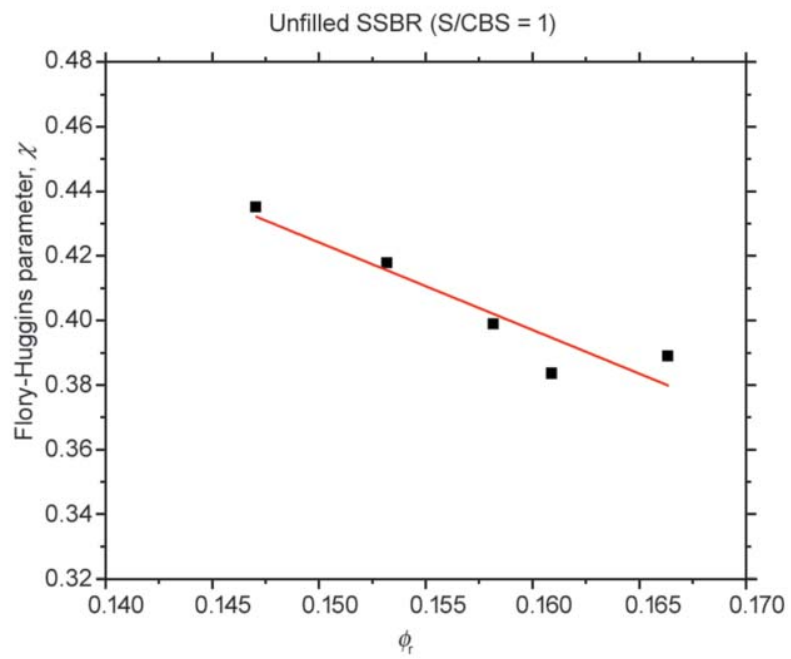

Figure 7. Estimated $\chi$ using the proposed calibration versus tube model fitting results of mechanical testing for unfilled SBR samples with a constant sulfur/accelerator ratio. assumption between mixing and elastic components of the free energy and the use of simplistic models of rubber elasticity [33]. The latter effect has been minimized by using the HHS approach; therefore the observed variation of $\chi$ is just a consequence derived from the failure of the mean-field assumption inherent to the Flory-Huggins model caused by the application of Flory-Rehner theory beyond its validity limit (e.g. large degree of swelling) [33].

Although the characteristic equations of the HHS model are of higher complexity than for the phantom and Flory-Rehner models, this can be overcome by reasonable approximations. In particular, in the case of moderately cross-linked networks (i.e. with strong topological constraints [13] and non-negligible crosslink density but far from the limits of highly crosslinked and entanglement dominated networks) which are of interest for technical applications, it is possible to use (see [16] and [20]) (Equation (11)):

$N_{\mathrm{c}} \approx N_{\mathrm{e}}$

$A_{\mathrm{c}} \approx 0.67$

$\frac{G_{\mathrm{N}}}{G_{\mathrm{c}}}=\frac{2}{\sqrt{6}} \cdot \frac{M_{\mathrm{c}}}{A_{\mathrm{c}} \cdot M_{\mathrm{e}}} \approx 1.219$

Using the approximations in Equation (11), the HHS model can be used for standalone evaluations of swelling experiments without the need of mechanical testing to obtain $A_{\mathrm{c}}, G_{\mathrm{N}}$ and $G_{\mathrm{c}}$. Using the unfilled NR samples as example, the difference between the $M_{\mathrm{c}}$ results of the calculations with the full model and its approximation (Equation (11)) is between 1 and $9 \%$ (taking as reference the original equation). Similarly, the comparison of the characteristic factor $B$ gives differences between 1 and $15 \%$ for the SBR samples and between 6 and $9 \%$ for the analyzed BR compounds. It should be noted that the appropriateness of the proposed approximated evaluation will depend on how much the sample differs from the condition $A_{\mathrm{c}} \approx 0.67$, which overall is a reasonable value to use in a standalone application of the HHS model.

\section{Conclusions}

In the present work, the application of the HHS model was exemplified for a limited set of data consisting of several groups of unfilled rubber samples in order to provide a first proof of the principle behind this approach and make the analytical equations for experimental data analysis available to the scientific community, which might stimulate a broader valida- 
tion of this model and its generalization to more complex (in particular) filled compounds. The application of the Helmis-Heinrich-Straube model as presented in this Letter can be used to address both the physical model that describes the elastic response of swollen networks and Flory-Huggins parameter uncertainties in swelling data treatment through a realistic network picture and a simple calibration process with mechanical properties, respectively.

The application of the HHS model will also be of benefit for deepening the understanding of several experimental approaches performed on swollen networks, like thermoporosimetry [34, 35], or more recently, time-domain DQ NMR experiments for quantifying the effective cross-link density [24, 26, 36]. This recently proposed procedure relies on the assumption that at large swelling degree, $Q$, it is possible to reach the so-named phantom reference state where the measured dynamic order parameter depends on the cross-links (and trapped entanglements active in swollen state) but not on entanglement packing effects. The obtained NMR results systematically underestimate $M_{\mathrm{c}}$ and overestimate $M_{\mathrm{e}}$ when they are compared to the structural parameters obtained by fits of stress-strain curves through the extended non-affine tube model [26]. These deviations have been related to an expected, but undefined, systematic error of the phantom reference network concept. Following the arguments of the HHS model and the experimental evidences shown in this work, it seems obvious that even in the high $Q$ regime the entanglement-related packing effects are not completely released and, consequently, their conformational constraints are measurable (e.g. NMR experiments $[24,26,36])$ and have to be taken into consideration for the data analysis. Finally, it is important to remark that the entanglement contribution to the elastic behavior of swollen rubber networks could not be applied as a simple rescaling factor because it is dependent on structural parameters (e.g. $M_{\mathrm{c}}, M_{\mathrm{e}}$ ) and, consequently, it could be different for each rubber matrix and network structure.

\section{Acknowledgements}

The authors thank Goodyear S. A. for the permission to publish this paper. The present project was supported by the National Research Fund Luxembourg (project ref. 6916525), whose financial support is acknowledged. JLV thanks the Ministerio de Ciencia e Innovación (Spain) for his Ramon y Cajal contract.

\section{References}

[1] Horkay F., McKenna G. B.: Polymer networks and gels. in 'Physical properties of polymers handbook' (ed.: Mark J. E.) Springer, New York, 497-523 (2007).

[2] Treloar L. R. G.: The physics of rubber elasticity. Clarendon Press, Oxford (2009).

[3] Frenkel J.: A theory of elasticity, viscosity and swelling in polymeric rubber-like substances. Rubber Chemistry and Technology, 13, 264-274 (1940). https://doi.org/10.5254/1.3539509

[4] Flory P. J., Rehner J.: Statistical mechanics of crosslinked polymer networks II. Swelling. Journal of Chemical Physics, 11, 521-526 (1943). https://doi.org/10.1063/1.1723792

[5] Flory P.: Statistical mechanics of swelling of network structures. Journal of Chemical Physics, 18, 108-111 (1950). https://doi.org/10.1063/1.1747424

[6] Flory P. J.: Principles of polymer chemistry. Cornell University Press, Ithaca (1953).

[7] Flory P. J.: Thermodynamics of high polymer solutions. Journal of Chemical Physics, 9, 660-661 (1941). https://doi.org/10.1063/1.1750971

[8] Huggins M.: Solutions of long chain compounds. Journal of Chemical Physics, 9, 440 (1941). https://doi.org/10.1063/1.1750930

[9] Valentín J. L., Carretero-González J., Mora-Barrantes I., Chassé W., Saalwächter K.: Uncertainties in the determination of cross-link density by equilibrium swelling experiments in natural rubber. Macromolecules, 41, 4717-4729 (2008). https://doi.org/10.1021/ma8005087

[10] James H. M., Guth E.: Theory of the elastic properties of rubber. Journal of Chemical Physics, 11, 455-481 (1943). https://doi.org/10.1063/1.1723785

[11] James H. M., Guth E.: Theory of the increase in rigidity of rubber during cure. Journal of Chemical Physics, 15, 669-683 (1947). https://doi.org/10.1063/1.1746626

[12] Helmis G., Heinrich G., Straube E.: Bestimmung der mittleren Netzbogenmolmasse von polymeren Netzwerken im Quellgleichgewicht (in German). Plaste und Kautschuk, 33, 53-55 (1986).

[13] Heinrich G., Straube E., Helmis G.: Rubber elasticity of polymer networks: Theories. Advances in Polymer Science, 85, 33-87 (1988).

https://doi.org/10.1007/BFb0024050

[14] Marckmann G., Verron E.: Comparison of hyperelastic models for rubber-like materials. Rubber Chemistry and Technology, 79, 835-858 (2006). https://doi.org/10.5254/1.3547969

[15] Xu B., Di X., McKenna G. B.: Swelling behavior of cross-linked rubber: Explanation of the elusive peak in the swelling activity parameter (dilational modulus). Macromolecules, 45, 2402-2410 (2012). https://doi.org/10.1021/ma202631u 
[16] Klüppel M., Heinrich G.: Network structure and mechanical properties of sulfur-cured rubbers. Macromolecules, 27, 3596-3603 (1994).

https://doi.org/10.1021/ma00091a022

[17] Erman B., Flory P. J.: Relationships between stress, strain, and molecular constitution of polymer networks. Comparison of theory with experiments. Macromolecules, 15, 806-811 (1982).

https://doi.org/10.1021/ma00231a023

[18] Kästner S.: Theorie der Polymernetzwerke mit behinderter Fluktuation I. Zustandsintegral, freie Energie und Frontfaktor bei behinderter Fluktuation der Vernetzungspunkte (in German). Colloid and Polymer Science, 259, 499-507 (1981).

https://doi.org/10.1007/BF01397887

[19] Heinrich G., Vilgis T. A.: Contribution of entanglements to the mechanical properties of carbon blackfilled polymer networks. Macromolecules, 26, 1109 1119 (1993).

https://doi.org/10.1021/ma00057a035

[20] Basterra-Beroiz B., Rommel R., Kayser F., Westermann S., Valentín J. L., Heinrich G.: New insights into rubber network structure by a combination of experimental techniques. Rubber Chemistry and Technology, 90, 347-366 (2017).

https://doi.org/10.5254/rct.16.83732

[21] Horkay F., McKenna G. B., Deschamps P., Geissler E.: Neutron scattering properties of randomly cross-linked polyisoprene gels. Macromolecules, 33, 5215-5220 (2000).

https://doi.org/10.1021/ma0003001

[22] Tan Z., Jaeger R., Vancso G. J.: Crosslinking studies of poly(dimethylsiloxane) networks: A comparison of inverse gas chromatography, swelling experiments and mechanical analysis. Polymer, 35, 3230-3236 (1994). https://doi.org/10.1016/0032-3861(94)90127-9

[23] Braun J. L., Mark J. E., Eichinger B. E.: Formation of poly(dimethylsiloxane) gels. Macromolecules, 35, 5273 5282 (2002).

https://doi.org/10.1021/ma0116046

[24] Chassé W., Lang M., Sommer J-U., Saalwächter K.: Cross-link density estimation of PDMS networks with precise consideration of networks defects. Macromolecules, 45, 899-912 (2012).

https://doi.org/10.1021/ma202030z

[25] Kaliske M., Heinrich G.: An extended tube-model for rubber elasticity: Statistical-mechanical theory and finite element implementation. Rubber Chemistry and Technology, 72, 602-632 (1999). https://doi.org/10.5254/1.3538822
[26] Syed I. H., Stratmann P., Hempel G., Klüppel M., Saalwächter K.: Entanglements, defects, and inhomogeneities in nitrile butadiene rubbers: Macroscopic versus microscopic properties. Macromolecules, 49, 9004 9016 (2016).

https://doi.org/10.1021/acs.macromol.6b01802

[27] Wagner H. L., Flory P. J.: Molecular dimensions of natural rubber and gutta percha. Journal of the American Chemical Society, 74, 195-200 (1952).

https://doi.org/10.1021/ja01121a050

[28] Bristow G. M., Watson W. F.: Viscosity-equilibrium swelling correlations for natural rubber. Transactions of the Faraday Society, 54, 1567-1573 (1958).

https://doi.org/10.1039/TF9585401567

[29] Marzocca A. J.: Evaluation of the polymer-solvent interaction parameter $\chi$ for the system cured styrene butadiene rubber and toluene. European Polymer Journal, 43, 2682-2689 (2007). https://doi.org/10.1016/j.eurpolymj.2007.02.034

[30] McKenna G. B., Flynn K. M., Chen Y. S.: Experiments on the elasticity of dry and swollen networks: Implications for the Frenkel-Flory-Rehner hypothesis. Macromolecules, 22, 4507-4512 (1989). https://doi.org/10.1021/ma00202a022

[31] McKenna G. B., Horkay F.: Effect of crosslinks on the thermodynamics of poly(vinyl alcohol) hydrogels. Polymer, 35, 5737-5742 (1994). https://doi.org/10.1016/S0032-3861(05)80049-7

[32] Horta A., Pastoriza M. A.: The interaction parameter of crosslinked networks and star polymers. European Polymer Journal, 41, 2793-2802 (2005). https://doi.org/10.1016/j.eurpolymj.2005.06.020

[33] Chassé W., Saalwächter K., Sommer J-U.: Thermodynamics of swollen networks as reflected in segmental orientation correlations. Macromolecules, 45, 5513-5523 (2012).

https://doi.org/10.1021/ma3009004

[34] Kuhn W., Peterli E., Majer H.: Freezing point depression of gels produced by high polymer network. Journal of Polymer Science, 16, 539-548 (1955). https://doi.org/10.1002/pol.1955.120168238

[35] Brun M., Lallemand A., Quinson J-F., Eyraud C.: A new method for the simultaneous determination of the size and shape of pores: The thermoporometry. Thermochimica Acta, 21, 59-88 (1977). https://doi.org/10.1016/0040-6031(77)85122-8

[36] Schlögl S., Trutschel M-L., Chassé W., Riess G., Saalwächter K.: Entanglement effects in elastomers: Macroscopic vs microscopic properties. Macromolecules, 47, 2759-2773 (2014). https://doi.org/10.1021/ma4026064 